\title{
Detecting network cyber-attacks using an integrated statistical approach
}

\author{
Benamar Bouyeddou, Fouzi Harrou, Benamar Kadri, Ying Sun
}

\begin{abstract}
Anomaly detection in the Internet of Things (IoT) is imperative to improve its reliability and safety. Detecting denial of service (DOS) and distributed DOS (DDOS) is one of the critical security challenges facing network technologies. This paper presents an anomaly detection mechanism using the Kullback-Leibler distance (KLD) to detect DOS and DDOS flooding attacks, including transmission control protocol (TCP) SYN flood, UDP flood, and ICMP-based attacks. This mechanism integrates the desirable properties of KLD, the capacity to quantitatively discriminate between two distributions, with the sensitivity of an exponential smoothing scheme. The primary reason for exponentially smoothing KLD measurements (ES-KLD) is to aggregate all of the information from past and actual samples in the decision rule, making the detector sensitive to small anomalies. Furthermore, a nonparametric approach using kernel density estimation has been used to set a threshold for ES-KLD decision statistic to uncover the presence of attacks. Tests on three publicly available datasets show improved performances of the proposed mechanism in detecting cyber-attacks compared to other conventional monitoring procedures.
\end{abstract}

Keywords TCP SYN flood. Smurf attack. KL distance . anomaly detection

\section{Introduction}

With the advancement in social media networks and computing technologies, and rapid development in the Internet of Things (IoT) technology, which connects a large number of digital devices, information becomes available from anywhere at any time and via a multitude of smart devices [1-3]. Unfortunately, network architectures, protocols, and hardware present many vulnerabilities that are eventually exploited by hackers to perform several types of attacks [4-6].

Denial of service (DOS) and distributed denial of service (DDOS) attacks have significantly increased and become a severe threat disrupting internet services. Generally speaking, DOS and DDOS flooding attacks inundate victims with a high rate of traffic to harm the network's infrastructures (e.g., servers and routers) and make them unavailable for legitimate users. Among the DOS and DDOS attacks, transmission control protocol (TCP) SYN flood, and internet control message protocol (ICMP) Smurf attacks are widely used by attackers to shut down systems and networks around

Benamar Bouyeddou and Benamar Kadri

STIC Lab., Department of Telecommunications, Abou Bekr

Belkaid University, Tlemcen, Algeria,

Email: bouben81@yahoo.fr

Fouzi Harrou and Ying Sun

King Abdullah University of Science and Technology

(KAUST), Computer, Electrical and Mathematical Sciences

and Engineering (CEMSE) Division, Thuwal, 23955-6900,

Saudi Arabia

Email: fouzi.harrou@kaust.edu.sa the world [7]. Moreover, most of the networks DOS and DDOS attacks against internet protocol version 6 (IPv6) networks are based on ICMPv6 protocol, such as ping flood, remote Smurf, router discovery, router solicitation, neighbor advertisement, and neighbor solicitation. Furthermore, both TCP and ICMP protocols are a key component in the current and future generation of networks, such as the IPv6 environment. That is why the detection of TCP-based and ICMP-based attacks is vital for network protection. Accordingly, this work focuses on developing an efficient detection mechanism to detect TCP SYN flood, Smurf attack, and ICMPv6-based DOS and DDOS attacks.

Several mechanisms were developed in the literature to prevent and protect networks from TCP SYN flood attacks [8,9]. In [10], a strategy integrating the intrusion detection system Snort with two commonly used software-defined networking (SDN) controllers, namely ODL (Opendaylaight) and ONOS (Open Networking system), is introduced. Besides the detection accuracy, which depends upon the predefined attack's signatures, the proposed tool is based on basic detection rules that can be easily manipulated by attackers. In [11], the quantum flow and hidden Markov chain are integrated to detect DDoS attack and C-Worm. Specifically, after measuring network traffic characteristics and constructing traffic profiles using the quantum flow, the hidden Markov chain is applied to reject anomalous traffics. The effectiveness of this method has been verified through 
simulated traffics obtained by using network simulator 2 (NS2). Results show that the number of established traffic profiles affect the resistance rate significantly. In [12], an OPEnflow-based REmedy (called OPERETTA) has been proposed to mitigate CP SYN flood attacks against web servers. However, the validation mechanism of this approach can cause a significant delay to legitimate requests. In [13], a monitoring mechanism called SLICOTs is designed based on forwarding TCP requests via temporary rules. Unfortunately, in addition to the delay caused by such rules, SLICOTs does not support flow aggregation and proactive-based rules networks. In [14], a classification system (CS)-DDOS is proposed to detect and prevent SYN flood in a Cloud environment. However, this approach assumes that the IP addresses are not spoofed, and the attackers are already blacklisted. In [5], a technique combining the stacked Non-symmetric Deep Auto-Encoder (NDAE) (deep learning) and the accuracy of the Random Forest (RF) algorithm (shallow learning) is introduced for intrusion detection. Results highlight the promising detection performance of this approach in detecting DOS and DDOS attacks. Nevertheless, the use of RF procedure makes the application of this approach challenging for real-time detection. In [15], a neural network-based detector called RBF-NN (Radial basis function neural network), which automatically configured through a Bat training algorithm, is introduced. Unfortunately, this detector is limited by the high dimensionality problem. Hence, an appropriate feature selection technique is needed to alleviate this difficulty. In [16], a packet trace-back method is introduced using the third party auditor (TPA) and Weibull distribution for analyzing the source of DDOS attacks in cloud computing. However, the distribution-based detection procedure requires large data, which makes it very challenging for real-time applications. In [17], a statistical class center-based triangle area vector approach is introduced to detect DOS attacks. This approach is proposed to bypass the problem of the conventional feature extraction-based detection methods by computing the mean of target classes individually and extracting the correlation between features. The Mahalanobis metric is applied for DOS attack detection. In [18], a method to detect DDOS attacks has been introduced based on an autonomous multi-agent system. In this method, the agents employ particle swarm optimization to achieve effective communication and good decision making. In [19], an innovative approach to detect DDOS attacks is proposed on the symmetrical uncertainty (SU)-Genetic feature selection. The SU-Genetic algorithm is applied to select the essential features of the original attack data. It has been shown that the performance of the conventional detection methods, including BayesNet, J48, and RanomTree, has been improved after using the SU-Genetic feature selection method.

During the last two decades, the ICMP protocol represented a vulnerable ingredient for attackers to initiate different types of cyber-attacks. Thereby, researchers have designed numerous detection procedures to protect networks against ICMP-based attacks. To tackle the ICMP-based attacks, the ICMPv4 messages (e.g., ping tool and broadcast service) were usually filtered in IPv4 networks, but with the coming of IPv6 protocol, this practice becomes no much longer valuable. Essentially, to deploy IPv6 networks, the ICMPv6 protocol must be implemented and activated [20]. In [21], Olszewski improved the Address Resolution Protocol watch (ARPwatch) used in IPv4 protocol by proposing the Neighbor Discovery Protocol Monitoring (NDPMon) to detect NDP-based attacks related to the ICMPv6 protocol in the IPv6 environment. Although it requires a long training step, this solution cannot detect duplicate address-based attacks and other forms of spoofing attacks (e.g., Router Advertisement and Neighbor Advertisement). In [22], a detection scheme applied the Shewhart chart to the Hellinger distance between the abnormal and normal traffics. Notably, this mechanism considers only normally distributed traffic to discriminate attacks. In [23], the detection of ICMPv6-based DOS and DDOS attacks is addressed as a binary hypothesis testing problem based on the Generalized likelihood ratio (GLR) test. The GLR is employed to decide between the null hypothesis (normal traffic) and the alternative hypothesis (abnormal traffic). In [24], another mechanism called Router Advertisement guard (RA guard) is proposed using routers and switches to control network traffic based on some characteristics of IPv6 packets, such as source address and interface. Nevertheless, this solution cannot be implemented on most commercialized switches nor used with a wireless access point. In [25], the multicast listener discovery (MLD) protocol is used to develop an IPv6 IDS. However, this solution can detect only attacks that are based on neighbor solicitation and neighbor advertisement messages. Other researchers proposed a collaborative system named FireCol using all internet service providers for ensuring network protection [26]. As a full distributed mechanism, however, it is very challenging to implement this solution in practice.

In this context, Kullback-Leibler (KL) divergence has been widely used in detecting many types of attacks occurring in different categories of networks [27-30]. In [31], Sahoo et al. compared the performance of general entropy (GE) and other information distances (ID) such as KL divergence to detect flooding DDOS in the SDN environment based on the destination IP address parameter. However, these techniques are designed based on the assumptions that the normal and the attack traffic have Gaussian and Poisson distributions, respectively. Also, the detection threshold is fixed manually through simulation. In [32], Li et al. presented a detection technique of injection attacks in cyber-physical systems using KullbackLiebler divergence. After numerous simulations on a three-tank system, it has been shown that the considered attacks have been significantly changed the system estimation and state. In [33], Zhang et al. 
applied Kullback-Leibler based detection to supervise the cyber-physical system and detect the stealthy deception attacks. In [34], an anomaly detection method called Ent-SVM is developed based on the information entropy and SVM algorithm. The information entropy is used to normalize traffic features, and SVM is applied for attack detection and classification. In [35], the authors discussed the efficiency of machine learning algorithms to classify attacks in cyber-physical systems correctly. They studied Support Vector Machine (SVM), K-Nearest Neighbor (KNN), and Naïve Bayes (NB) under different feature selection methods. Detection results showed that using a small set of features improves the computation time but can significantly degrade the detection accuracy. In [36], three supervised machine learning methods, namely neural network, SVM, and $\mathrm{NB}$, have been applied to identify cyber-attacks in a modern power system. It has been underlined that machine learning methods have good potential in cyber-attacks detection in critical cyber-physical infrastructures. However, supervised detectors require the availability of labeled data in training, which is not often accessible. In [37], an intrusion detection method is proposed to detect and discriminate various types of Building Automation and Control network (BACnet) attacks in Building Automation Systems (BAS) networks. This method is designed using the concept of Context Awareness and Anomaly Behavior Analysis (ABA), and its detection performance has been assessed using Smart Building testbed developed at the University of Arizona Center for Cloud and Autonomic Computing. Results show the promising performance of this method in detecting BACnet attacks. In [38], an efficient technique is considered for modeling the behavior of the Internet of Things (IoT) devices and detecting the results of attacks against their protocols like Wi-Fi and Bluetooth. Experimental results demonstrate that the anomaly behavior analysis-based intrusion detection systems provide good detection performance for the Wi-Fi and the Bluetooth protocols.

However, it is worth noticing that the DOS and DDOS detection methods based on the KLD metric aforementioned are designed based on the normality assumption of traffic distribution. Accordingly, as traffic data collected from networks are often nonnormally distributed, this leads to misleading anomaly detection. Also, the detection thresholds in the conventional KLD-based techniques are manually fixed or not used at all in the detection process. Thus, the main goal in this paper is to extend the advantage of the KLD-based monitoring approach to handle nonGaussian processes and improve the detection of DOS and DDOS attacks.

Thereby, this paper introduces an effective detection mechanism based on KLD to detect TCP SYN flood, UDP flood, ICMP Smurf attacks, and attacks related to the ICMPv6 version. Indeed, KLD measures the mismatch between two probability distributions, which make it very promising in developing anomaly detection techniques [39]. Due to its high sensitivity, KLD can be used to reveal even small deviations from the normal behavior of the monitored network. Hence, potential attacks can be detected by monitoring the distance between distributions of newly captured traffic packets (suspicious or anomalous) and the training traffic (i.e., attacks-free traffic). An additional novelty in the proposed approach, to further improve the detection performance we applied an exponential smoothing to the KLD sequences. The major reason for exponentially smoothing KLD measurements (ES-KLD) is to include all of the information from past and actual samples in the decision rule, which makes it sensitive to small anomalies. Furthermore, a nonparametric approach using kernel density estimation has been used to set a threshold for ES-KLD decision statistic, which extends the flexibility of the developed method. To verify the efficiency of the proposed schemes, we compare the obtained results to those of state of the art approaches using quantitative metrics, namely, true positive rate (TPR), false-positive rate (FPR), Accuracy, and area under the curve (AUC) [40]. Experiments are carried out using three different traces of IP networks traffic, the first is the 1999 DARPA Intrusion Detection Evaluation dataset, the second is the MAWI dataset and the third one is the ICMPv6 dataset.

The remainder of this paper is organized as follows. Section 2 describes the TCP SYN flood, the ICMP Smurf, and ICMPv6-based flooding attacks. In section 3, we give brief details about the ES-KLD-based anomaly detection mechanism. Section 4 evaluates the capacity of the ES-KLD approach to uncover different types of DOS and DDOS attacks using three datasets. Finally, conclusions and discussions are presented in section 5.

\section{DOS and DDOS flooding attacks}

\subsection{TCP SYN flood attack}

In recent years, TCP SYN flood attacks are often launched by attackers to perform DOS/DDOS to shutdown systems, networks, and servers. A normal TCP connection (Fig. 1(a)) is established in three steps (three-way handshake). Specifically, three messages are transmitted to negotiate and start the TCP session before any data exchange between a client and a server. (1) The client sends to the server a synchronization (SYN) segment for requesting a new connection. (2) The server responds with a single segment that contains the client's SYN acknowledgment SYNchronization/ACKnowledgement (SYN/ACK). The server also maintains this request, half-open, in the backlog queue. (3) Finally, the client acknowledges the received SYN/ACK with the ACK segment. Now, the connection is made, then the server removes it from the backlog queue, and the client can send its data. Unfortunately, TCP SYN flood attacks (Fig. 1(b)) can generate a massive number of half-open connections that exhaust the server's backlog queue and make it incapable of handling new TCP sessions, including 
incoming requests from legitimate clients. Generally, to perform a TCP SYN flood attack, the malicious users can either do not send the ACK segment or using a spoofed IP address [41].
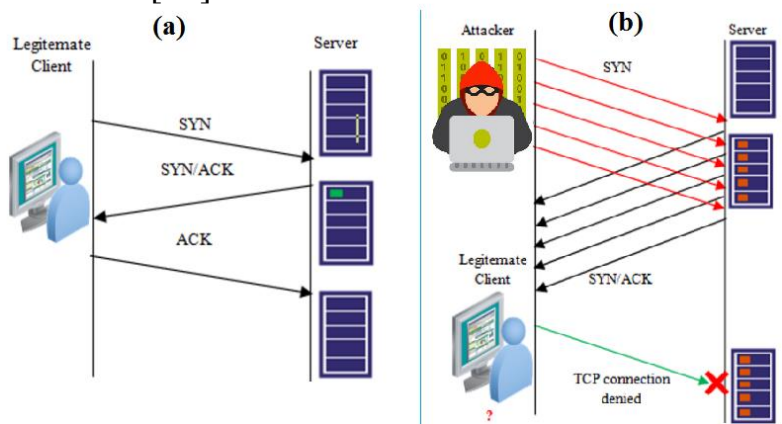

Fig. 1 (a) Establishment of normal TCP connection, and (b) TCP SYN flood attack

\subsection{ICMP Smurf attacks:}

The TCP/IP architecture implements the ICMP protocol to transmit administration messages (e.g., Traceroute and Ping) and error messages, such as destination network/host/protocol unreached, time to live expired, fragmentation required, and redirect messages. However, ICMP messages are frequently exploited by attackers to conduct several types of DOS/DDOS attacks (e.g., ping of death, ping flood, and Smurf) [20]. The ICMP amplification attacks, also known as Smurf attacks, are pertinent types of DDOS attacks, based either on ICMPv4 or the ICMPv6 versions, menace IPv4 as well as IPv6 technology [42]. Indeed, the Smurf attacks use broadcast servers to overwhelm their victims with a high rate of ICMP EchoReply messages. To accomplish such an attack, the attacker starts by spoofing the targeted victim's IP address, then sending ICMP Echo Request messages to the broadcast server, which delivers them to all hosts (Fig. 2). These last respond with the ICMP Echo-Reply message to the victim's IP address. A broadcast network can contain hundreds of hosts; hence, the victim will be flooded by many Echo-Reply messages that exhaust its resources and turn it out of service [20].

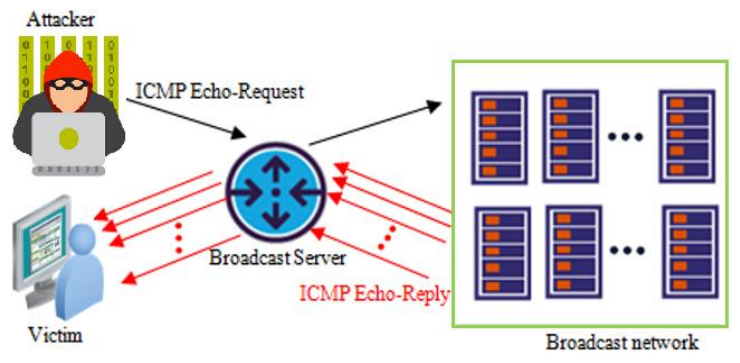

Fig. 2 Illustration of ICMP amplification attack (i.e., Smurf attack)

\subsection{ICMPv6-based DOS and DDOS attacks}

The ICMPv6 protocol can be considered the key element in IPv6 networks. It is used to run the basic functionalities such as Neighbor Discovery, Solicitation and Advertisement, Router Solicitation, and Advertisement. To accomplish all these tasks, the ICMPv6 protocol provides numerous messages but without any mechanism of protection about their generation, transmission, and reception. This lack of security makes it vulnerable to different types of cyberattacks, including several DOS and DDOS attacks, such as ICMPv6 ping flood, ICMPv6 smurf, router discovery, and neighbor discovery floods. In IPv6 networks, router discovery operation permits to any host to determine routers that are located in its subnet and eventually to use them later to forward its data. To this end, hosts multicast an ICMPv6 message called Router Solicitation (RAS) requesting a valid link (Fig. 3). The nearest router responds with the ICMPv6 message Router Advertisement (RAD) that includes all needed routing information. The neighbor discovery enables two connected devices on the same network to discover and exchange their configuration information. The neighbor discovery is achieved through two ICMPv6 messages: Neighbor Solicitation (NAS) and Neighbor Advertisement (NAD). Attackers can exploit the RAD, RAS, and NAS messages in many ways to perform a different kind of DOS and DDOS attacks. The ICMPv6-based flooding is a common type of such attacks, where a large amount of forged messages is transmitted to overwhelm the victim and turn it out of service [42].

\section{Proposed ES-KLD monitoring scheme}

As discussed above, an integrated ES-KLD-based anomaly detection mechanism is proposed to detect DOS and DDOS flooding attacks. The proposed method is based on the exponentially smoothing of the KLD measurements. Indeed, the KLD allows us to quantify the deviation of the captured traffic that possibly includes DOS and DDOS attacks from the normal (i.e., attacks-free) traffic. When the exponentially smoothed KLD between captured traffic and the attack-free traffic is relatively large and exceeds a predefined threshold, the presence of DOS or DDOS attacks is flagged.

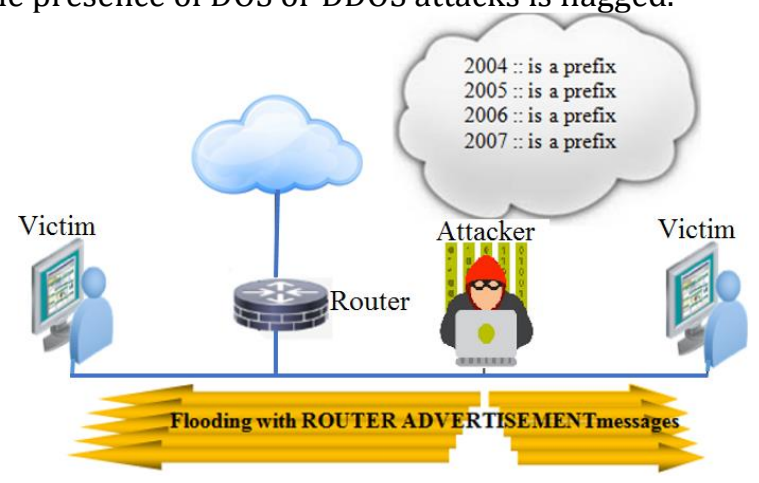

Fig. 3 Example of an ICMPv6 DOS attack based on the RAD message 


\subsection{KLD metric}

The KLD originates from the information theory domain and is actually present on board of many disciplines such as classification, speech and image recognition [43, 44], transportation [39], industry [45], [46], and medicine [47]. The KLD computes the distance between two probability distributions. The Kullback-Leibler information between two probability density functions (PDFs) $p_{1}(x)$ and $p_{2}(x)$ is defined as

$$
I\left(p_{1}: p_{2}\right)=\int_{\mathbb{R}^{d_{x}}} p_{1}(x) \log \left[\frac{p_{1}(x)}{p_{2}(x)}\right] d x,
$$

and between $p_{2}(x)$ and $p_{1}(x)$ is given by

$$
I\left(p_{2}: p_{1}\right)=\int_{\mathbb{R}^{d_{x}}} p_{2}(x) \log \left[\frac{p_{2}(x)}{p_{1}(x)}\right] d x,
$$

which is nonsymmetric (i.e $I\left(p_{1}: p_{2}\right) \neq I\left(p_{2}: p_{1}\right)$ and nonnegative (i.e $I\left(p_{1}: p_{2}\right) \geq 0$ and $I\left(p_{2}: p_{1}\right) \geq 0$ ). The KLI is a non symmetric measure, which means that $I\left(p_{1}: p_{2}\right)$ is not equal to $I\left(p_{2}: p_{1}\right)$. The KLD distance represent the symmetric form of KLI [45]. For the two distributions $p_{1}(x)$ and $p_{2}(x)$, the KLD metric is expressed as:

$$
\operatorname{KLD}\left(p_{1}: p_{2}\right)=I\left(p_{1}: p_{2}\right)+I\left(p_{2}: p_{1}\right)
$$

For two Gaussian distributions $p_{1} \sim \mathcal{N}\left(\mu_{0}, \sigma_{0}\right)$ and $p_{2} \sim \mathcal{N}\left(\mu_{1}, \sigma_{1}\right)$, characterized respectively by their means $\mu_{0}$ and $\mu_{1}$ and variances $\sigma_{0}^{2}$ and, $\sigma_{1}^{2}$, the KLD distance have the following analytical expression [48]:

$$
\begin{aligned}
& K L D\left(p_{1} \backslash \backslash p_{2}\right)= \frac{1}{\sigma_{0} \sqrt{2 \pi}} \int \exp \left(\frac{\left(x-\mu_{0}\right)^{2}}{2 \sigma_{0}^{2}}\right) \\
& {\left[\log \frac{\sigma_{1}}{\sigma_{0}}-\frac{\left(x-\mu_{0}\right)^{2}}{2 \sigma_{0}^{2}}+\frac{\left(x-\mu_{1}\right)^{2}}{2 \sigma_{1}^{2}}\right] d x } \\
&=\frac{\left(\mu_{1}-\mu_{0}\right)^{2}}{2 \sigma_{1}^{2}}+\frac{1}{2}\left(\log \frac{\sigma_{1}^{2}}{\sigma_{0}^{2}}+\frac{\sigma_{0}^{2}}{\sigma_{1}^{2}}-1\right) .
\end{aligned}
$$

The KLD measurements will be negligible if $p_{1}(x)$ and $p_{2}(x)$ are too close and become important when the two distributions are not similar. Hence, KLD can be utilized to reveal anomalies in network traffic. Specifically, when a network runs out of attacks, small values of KLD are expected. Else, high values arise if the network is under attack or in the presence of other anomalies.

\subsection{Kernel density estimation (KDE)}

In statistics, the kernel density estimation $\mathrm{KDE}$ is one of the most effective tools which is commonly used to estimate the probability distribution of a given data. For $\mathrm{X}=x_{1} ; \cdots ; x_{n}$ observations, KDE gives a nonparametric estimation of the probability density function (PDF) by the following formula [49]:

$$
p(x)=\frac{1}{n h} \sum_{i=1}^{n} K\left(\frac{x-x_{i}}{h}\right),
$$

where $x_{i}$ is the ith observation. $\mathrm{K}$ is the kernel function. Among a lot of kernel functions, the Gaussian kernel is usually used [50]:

$$
K(x)=\frac{1}{2 \pi} \exp \left(\frac{-\theta^{2}}{2}\right),
$$

$h$ represents the smoothing bandwidth factor that determines the probability estimation quality. Small values lead to undersmoothed PDFs. On the other hand, large values over-smooth them. As shown in [51], for $n$ observations with the standard deviation $\sigma$, the optimal choice of $h$ can be obtained from the following expression: $h=1.06 \sigma n^{-0.2}$.

\subsection{ES-KLD approach}

This section briefly describes the ES-KLD anomaly detection mechanism. The core idea of this approach consists of computing the KLD metric between the PDFs of the captured traffic and attack-free traffic (training data). Intuitively speaking, in cases of attackfree traffic, KLD is close to zero and in the presence of attacks, KLD deviates significantly from zero indicating the presence of an abnormal event. In the proposed mechanism, KLD sequences are exponentially smoothed to further improve its sensitivity to abnormal events (attacks). The variables used as the input to the proposed detection mechanism are mainly the variables characterizing the traffic behavior (e.g., number of bytes, number of packets, and IP address), which are extracted from different protocols, such as TCP, UDP, and ICMP. For instance, to detect SYN flood attacks, the observations represent the number of all TCP segments or only the received SYN segments par sampling time. Let's define the sequence of KLD measurements as computed in (3): $\operatorname{KLD}=\left[d_{1} \cdots d_{n}\right]$. The ES-KLD statistic is computed as:

$$
z_{t}^{K L D}=v d_{t}+(1-v) z_{t-1}^{K L D},
$$

where the initial value, $z_{0}^{K L D}$ is the anomaly-free mean of KLD vector, $\mu_{0}^{K L D}$. $v(0<v \leq 1)$ is a smoothing parameter.

Importantly, from (7) we note that the ES-KLD decision statistic incorporates the information from the past and current observations in the decision process, which helps improving its sensitivity to small changes. To explicitly demonstrate this point, the ES-KLD is recursively expressed as:

$$
\begin{gathered}
z_{t}^{K L D}=v d_{t}+(1-v)\left[v d_{t-1}+(1-v) z_{t-2}^{K L D}\right] \\
=v d_{t}+v(1-v) d_{t-1}+(1-v)^{2} z_{t-2}^{K L D}
\end{gathered}
$$

Using equation (7) recursively, we get :

$$
\begin{gathered}
z_{n}^{K L D}=v d_{n}+v(1-v) d_{n-1}+v(1-v)^{2} d_{n-2}+\cdots \\
+v(1-v)^{n-1} d_{1}+v(1-v)^{n} d_{0}
\end{gathered}
$$

Equation (9) can also be expressed in compact form as: 


$$
z_{t}^{K L D}=v \sum_{t=1}^{n}(1-v)^{n-t} d_{t}+(1-v)^{n} d_{0},
$$

where $v(1-v)^{n-t}$ represents the weight for $d_{t}$, which decreases exponentially for past samples. Essentially, the value of $v$ is practically selected between 0.2 and 0.3 for uncovering small changes.

We define a nonparametric detection threshold as the (1- $\alpha)$-th quantile of the estimated distribution of ES-KLD statistic, $z^{K L D}$, using kernel density estimation (KDE). An anomaly is detected when the ES-KLD decision function overpass the detection threshold. The general framework of the ES-KLD proposed approach is illustrated in Fig. 4.

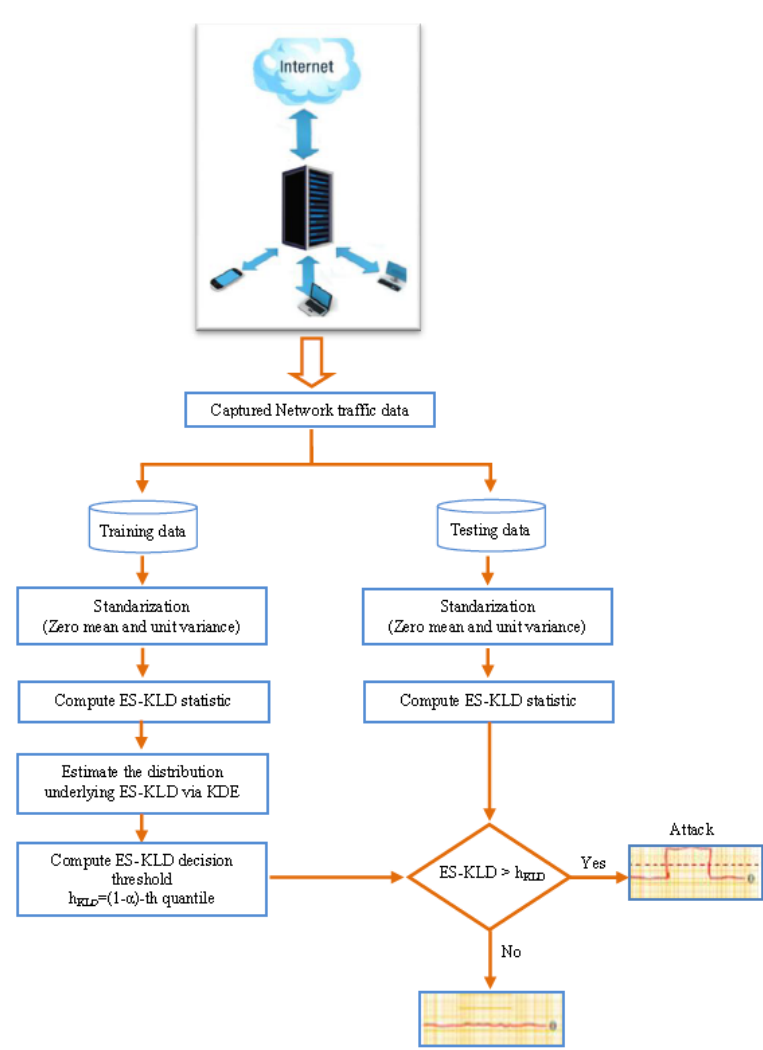

Fig. 4 Conceptual schematic of the ES-KLD detection approach

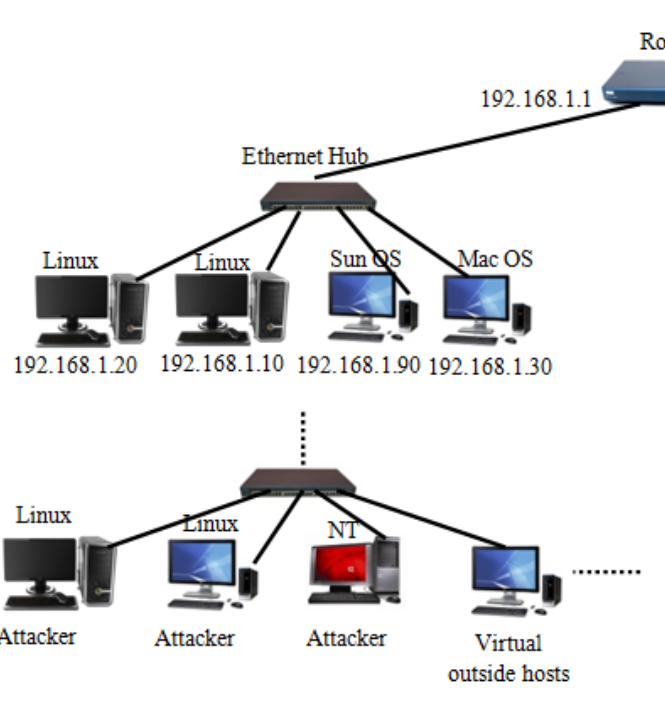

Fig. 5 Topology of DARPA 99 network

Using the Kernel density estimator (KLD) allows us to effectively deal with the high dynamicity of traffics and their derived characteristics and the need to estimate them continuously. The computational complexity of KDE is quadratic in the number of observations. Nevertheless, appropriate sampling of the dataset can reduce it significantly. Specifically, it can be reduced significantly through different methods including kernel merging, sampling, and space portioning, or by means of a practical sampling of datasets. With a sampling time of 10 s, enough observations can accurately estimate the underlying distributions. Therefore, this tradeoff makes ES-KLD more flexible and adapted even to the constrainedresources (e.g., storage, processing capacities, and energy) environments (e.g., IoT, and wireless networks). In centralized or distributed systems, it can be implemented as a host or network-based detection technique in which no exchanges between the network's components are required. In practice, the most challenging point is in evolutionary systems. In this case, the detection threshold can be automatically re-established as needed to go along with the system evolution. 


\section{Experiment results}

Now, the ability of the proposed ES-KLD mechanism in identifying some types of DOS and DDOS attacks is assessed. Precisely, the TCP SYN flood, UDP flood, Smurf, and ICMPv6-based attacks will be studied. To this end, experiments using IP network traffic from three databases are conducted.

\subsection{MAWI dataset}

The MAWI (Measurement and Analysis on the WIDE Internet) dataset is real internet traffic provided by the MAWI Working Group Traffic repository. In this dataset, the traffic is captured from many trans-pacific links (i.e., sampleponit-A, sampleponit-C, sampleponit$D$, and sampleponit-F) between the Japanese WIDE network and USA. The sampleponit-F, which is the most used, provides a daily trace of 15 minutes (min). In this study, TCPDUMP trace of January, 1st, 2010; $14 \mathrm{~h} 00$ to $14 \mathrm{~h} 15$ are used

(http://www.fukuda-lab.org/mawilab/data.html).

\subsection{DARPA99 dataset}

The DARPA 99 data is provided by Lincoln Laboratory at Massachusetts Institute of Technology (MIT) under the sponsorship of the defense advanced research projects agency (DARPA) and air force research laboratory. This data is generated using a simulation network mimicking a real network of a US air force base. Fig. 5 displays the configuration of this network. The training data comprises three weeks of anomalyfree traffic and the testing data comprises two weeks with different types of attacks (https://www.ll.mit.edu/ideval/data/1999data.html).

\subsection{ICMPv6 traffic dataset}

ICMPv6 traffic dataset is generated using the network presented in Fig. 6. The data without anomalies consists of 48 hours of ICMPv6 traffic. The anomalies that comprise numerous ICMPv6-based DOS and DDOS flooding attacks are generated based on the THC toolkit [52].

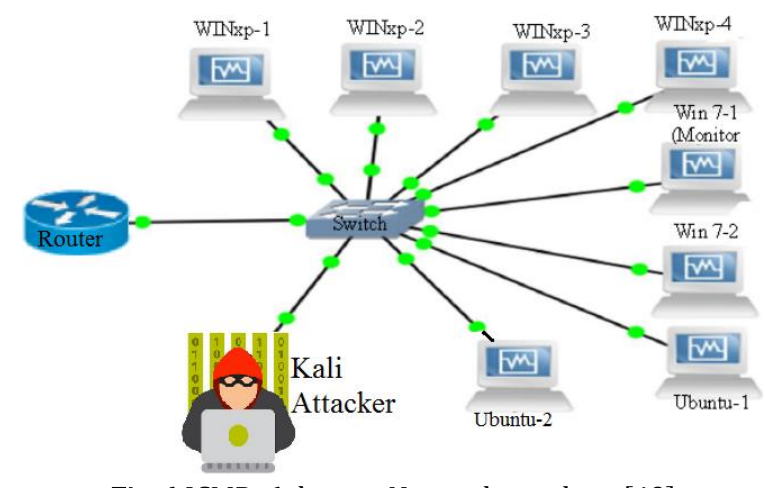

Fig. 6 ICMPv6 dataset Network topology [49]

\section{DETECTION RESULTS}

\subsection{Datasets preprocessing and DOS/DDOS flooding attacks detection using ES-KLD}

Here, we assess the performance of ES-KLD approach in detecting different types of DOS/DDOS flooding attacks by monitoring features from different protocols.

- $\quad$ TCP SYN flood attacks are monitored based on the flow of SYN segments and TCP flow received per sampling time.

- UDP flood attacks are inspected using the numbers of UDP datagram and ICMP destination unreachable messages.

- Ping flood attacks are detected using the received ICMP echo request messages and the whole ICMPv4 traffic.

- Smurf attacks are monitored based on the number of the received ICMP echo reply messages and the whole ICMPv4 traffic.

- ICMPv6-based flooding attacks are detected by checking NAD, NAS and RAD messages. In this case, we control the flow of these messages and the whole ICMPv6 traffic as well.

Here, to extract the relevant features (e.g., TCP SYN segments and TCP flow) from network traffic datasets, a prepossessing of the three datasets is performed using Wireshark (https://www.wireshark.org) and MySQL tools.

\subsection{Detection of DARPA99's DOS flooding attacks}

The DARPA99 dataset provides different types of DOS flooding attacks including TCP SYN flood, UDP flood, and ICMP Smurf attacks. In this work, we investigate the detection of the following attacks, which are recapitulated in Table 1. (i) Three TCP SYN flood attacks that run in the fifth week (Table 1). (ii) Two 15min UDP flood attacks are generated in week 5 day1 at 20:00:27 pm against two different victims. (iii) Five ICMP Smurf attacks, which have targeted the same victim during the fourth and fifth weeks. The beginning was with the week 4 , in which two attacks of 1 s were initiated on day 1 at 21:34:16 pm and 21:34:26 pm, one attack of $1 \mathrm{~s}$ at 18:29:25 pm in day 3 and another attack of $2 s$ at 08:45:18 am on day 5. Finally, the victim was re-attacked again in week 5, day 1 at 09:33:00 am during $2 \mathrm{~min}$. In detection results, we refer to the traffic of the ith Day in the ith week as WiDi (e.g., W5D1 to say Week 5 Day 1). Fig. 7 (a-b) shows the results of the ESKLD mechanism based on the received SYN messages. Firstly, the detection results confirm the high sensitivity of ES-KLD to changes. Accordingly, small values of ES-KLD statistics ( 0 or close to 0 ) are obtained when the monitored traffic is free from attacks. Else, with the appearance of DOS attacks, the anomalous traffic becomes too different from the normal attackfree traffic, so ES-KLD statistics increase significantly and large values are recorded. Also, small detection 
thresholds are established, which means that ES-KLD can reveal even low rate DOS and DDOS attacks. In Fig. $7(\mathrm{a})$, which represents the detection based on the traffic of W5D1, the attack happened from instances 605 to 611. During this attack, the victim (@IP=172.16.112.50) has received an average of 2928 SYN segments per observation time. According to Fig. 7(b), there are two attacks on the traffic of W5D2. In the first attack, the victim was overwhelmed by an average of 3027 SYN/observation time from instances 219 to 232. In the second attack, the victim 192.168.1.1 was inundated by 10256 SYN segments from instance ranged between 617 and 620. Fig. 7 (a-b) indicates that the SYN flood attacks are successfully detected and the detection rate of the ES-KLD mechanism has reached $100 \%$. In both attacks, it is clearly illustrated that the ES-KLD statistics increase proportionally to DOS attack intensity.

Table 1 SYN flood, UDP flood and Smurf attacks in the DARPA99 dataset

\begin{tabular}{cccccc}
\hline \multicolumn{2}{c}{ Attack } & $\begin{array}{c}\text { Wee } \\
\mathrm{k}\end{array}$ & Day & $\begin{array}{c}\text { Time of } \\
\text { appearance }\end{array}$ & Duration \\
\hline \multirow{2}{*}{ TCP SYN } & Attack 1 & 5 & 1 & $18: 04: 04$ & $6 \mathrm{~min} 51 \mathrm{~s}$ \\
flood & Attack 2 & 5 & 2 & $11: 48: 42$ & $1 \mathrm{~s}$ \\
& Attack 3 & 5 & 2 & $18: 16: 05$ & $3 \mathrm{~min} 26 \mathrm{~s}$ \\
UDP & Attack 1 & 5 & 1 & $20: 00: 27$ & $15 \mathrm{~min}$ \\
flood & Attack 2 & 5 & 1 & $20: 00: 27$ & $15 \mathrm{~min}$ \\
& Attack 1 & 4 & 1 & $21: 34: 16$ & $1 \mathrm{~s}$ \\
ICMP & Attack 2 & 4 & 1 & $21: 34: 26$ & $1 \mathrm{~s}$ \\
Smurf & Attack 3 & 4 & 3 & $18: 29: 25$ & $1 \mathrm{~s}$ \\
& Attack 4 & 4 & 5 & $08: 45: 18$ & $2 \mathrm{~s}$ \\
& Attack 5 & 5 & 1 & $09: 33: 00$ & $2 \mathrm{~min}$ \\
\hline
\end{tabular}

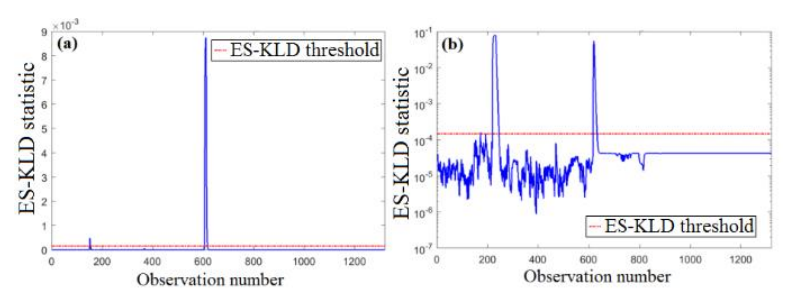

Fig. 7 ES-KLD results in the presence of SYN flooding attack in (a) W5D1 SYN Messages and (b) W5D2 SYN messages

Furthermore, Fig. 7 (a) indicates that the ES-KLD mechanism reveals another abnormal event at instances 150 and 151. Indeed, during these two instances, many TCP connections are requested from different clients excepting the owner of the IP address 202.77.162.213 that sent to 172.16 .114 .50 (hosting web servers) 1007 and 561 SYN segments at instances 150 and 151, respectively. According to the authors of the DARPA 99 dataset, 20 SYN segments sent simultaneously can shutdown the victim. Accordingly, the traffic in these two instances has certainly an anomalous behavior that can be considered as abnormal events, missed to be reported by the authors of the dataset. These anomalies can be classified as one of the following attacks: SYN flood, port sweep, port scan, or Network Mapper (NMAP). However, referring to the dataset documentation and their attacking procedure, the attacker sends 20 SYN segments to each port of the victim. After analyzing the raw W5D1 Tcpdump file, it noted that the source neither follows that procedure in sending its SYN segments nor runs the port sweep and port scan attacks. Here, the client 202.77.162.213 has sent all SYN segments to port 80 of 172.16.114.50, thus we can conclude that it is not an attack. But, it remains abnormal traffic with a peak of 39 SYN segments/s. Also, in Fig. 7 (b), a peak at instance 171 exceeds the established decision threshold. In this scenario, SYN segments generation does not follow the authors' attacking procedure. Specifically, the user with IP address 172.16 .112 .207 sent in 1 second about 27 SYN segments to port 80 of the IP address 206.246.131.226 and the client using the IP address 172.16.115.5 sent continuously in 4 seconds 73 and 48 SYN segments to the port 80 of 207.18.199.3 and 209.67.29.11, respectively. Here, it can be noticed that (i) there are some errors in attack's labeling (e.g., UDP flood attack in W5D1 and Smurf attack in W4D5 are missed). (ii) There are some critics about the ground traffic (i.e., normal traffic) that can strongly affect the training data; the network presents a very low activity, even seems in some traces runs out of service. Now, the performances of the ES-KLD mechanism in detecting TCP SYN flooding attacks are investigated when the whole TCP flow is monitored. Fig. 8 (a-b) displays the detection results of the ES-KLD mechanism. Although the attacks are detected, it can be noticed that monitoring all TCP flow cannot necessarily lead to good detection accuracy (i.e., it is not a good indicator of TCP SYN flooding attacks). As a connected protocol, different segments (e.g. Acknowledgment, Push, and Reset) are used to maintain the quality of the established sessions. Indeed, these segments can have a high contribution to the whole TCP flow and thus can strongly affect the detection of TCP SYN attack. In Fig. 8 (a), they (i.e., Acknowledgment, Push, and Reset segments) represent $84.2 \%, 93.12 \%$, and $95.07 \%$ of all TCP flow at the instances 150, 366 and 367, respectively. They can even mask attacks in some situations, particularly in flash crowd events and bad link quality in which more Acknowledgments and retransmission demands will be transmitted. The SYN attack is hardly detected; the number of SYN segments represents less than $35 \%$ of TCP flow in the instance 605 and around 41\% at the instances 606 and 607.

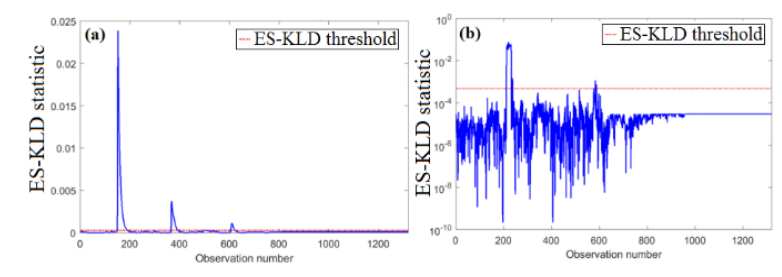

Fig. 8 ES-KLD results in the presence of TCP SYN flooding attack on (a) W5D1 TCP flow and on (b) W5D2 TCP flow

Fig. 9 illustrates the detection results of the ES-KLD mechanism in the presence of UDP flood attacks. Fig. 9 
indicates that these attacks have been successfully detected. During these attacks, the victims (@IP 172.16.112.50 and 172.16.113.50) have exchanged between them 272742 UDP datagram. Specifically, each one has sent 136371 UDP datagram to the Echo port of the other. We can observe that, during the two attacks, the ES-KLD statistics exceed largely the detection threshold. In Fig. 9, we also observe the presence of some peaks that begun at the instance 45. During these instances, the user with address IP 172.16.112.100 has continued sending DNS queries (a total of 12472 queries) even though the DNS server (@IP 172.16.112.20) indicates that the requested names are not available. Fig. 10 (a-d) displays the detection results of the ES-KLD mechanism when applied to the received Echo Replay messages in the case of Smurf attacks. All considered attacks are flagged by the designed mechanism (Fig. 10 (a-d)).

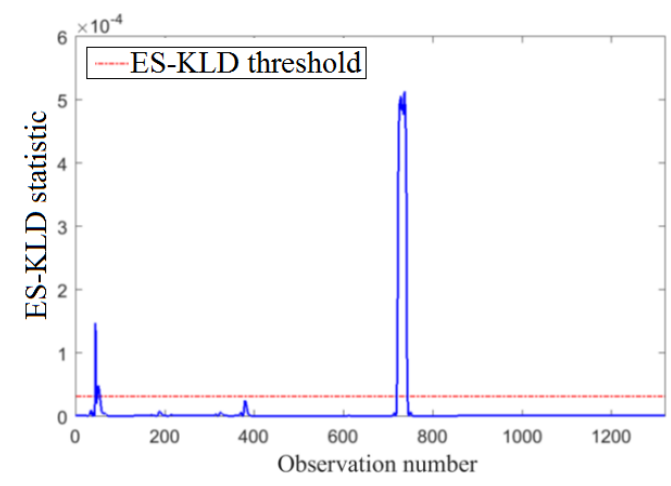

Fig. 9 ES-KLD results in the presence of UDP flood Dos attack on W5D1

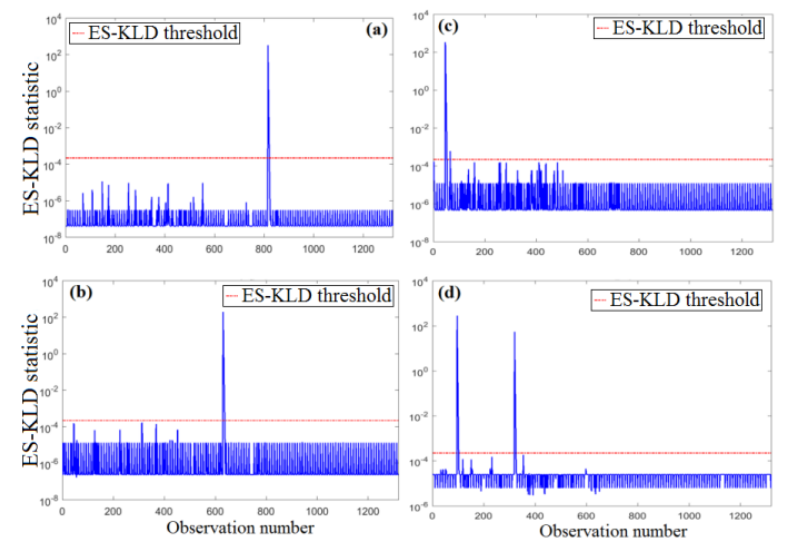

Fig. 10 ES-KLD results in the presence of abnormal ICMP echo reply messages on (a) W4d1, (b) W4d3, (c) W4d5 and (d) W5d1

In Fig. 10 (a), the Smurf attacks happened at the instance 815, and their victim (@IP 172.16.112.50) was inundated with 51681 Echo Reply messages. Fig. 10 (b) shows that the traffic W4D3 includes a Smurf attack, at instance 630, in which the targeted victim (@IP 172.16.112.100) has received 4455 Echo Reply messages. In Fig. 10 (c), the Smurf attack of W4D5's traffic occurred at instance 46, where 4453 Echo Reply messages were sent to the victim (@IP 172.16.112.50).
Fig. 10 (d) reveals two attacks in the W5D1 traffic. The first attack targeted the @IP 172.16.112.50 at instance 95 with 6000 Echo Reply messages, and the second attack was against the @IP 172.16.114.50 at instance 319 with 2655 Echo Reply messages.

Fig. 11(a-d) shows the detection results of the ESKLD algorithm when applied to ICMP traffic flow in the presence of Smurf attacks. As shown in Fig. 11(a-d), the results indicate that ICMP traffic can be used to detect the Smurf attacks. However, it can generate false alarms due to other ICMP's messages incorporated in the ICMP traffic. In Fig. 11(b-c), the destination and unreachable port messages are responsible for these two anomalies in W4D3 and the three anomalies in W4D5 traffic where they represent $100 \%$ of the ICMP traffic. In Fig. 11(c), during the two instances 307 and 308 , there are 267 destination unreachable messages, while any Echo Reply message was generated. Also, the Echo Reply messages represent $0 \%$ of the ICMP traffic (Fig. 11(d)) at the instances 178,179 and 352, although it counts 192,188 and 167 messages, respectively. Table 2 summarizes the detection performance of the proposed ES-KLD mechanism in terms of the true positive rate (TPR), false-positive rate (FPR). From Table II, it can be seen that the ES-KLD algorithm provides satisfactory performance in detecting DARPA99's DOS flooding attacks with small FPR and high TPR. This confirms the promising detection efficiency of the ES-KLD mechanism. The performance degradation noticed in TCP SYN flood (attack 2), and UDP flood (attack1) are in respect to DARPA's dataset specifications. Else, other anomalies missed by the authors are revealed, and as a result, higher TPR and lower FPR will be obtained.

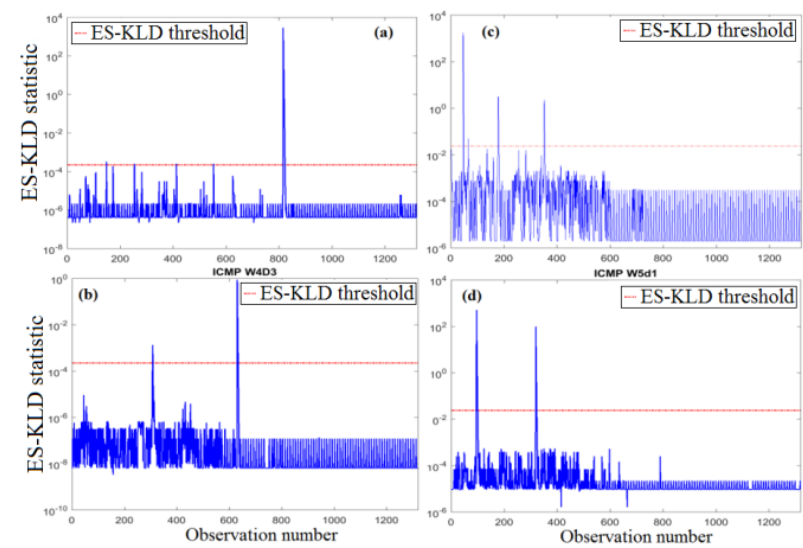

Fig. 11 ES-KLD results in the presence of Smurf attack (ICMP flow) on (a) W4D1, (b) W4D3, (c) W4d5, and (d) W4d5

Table 2 ES-KLD detection performance when using DARA99 dataset

\begin{tabular}{cccccc}
\hline \multicolumn{2}{c}{ Attack } & TPR & FPR & Accuracy & AUC \\
\hline TCP SYN & Attack 1 & 1 & 0.002 & 0.998 & 0.999 \\
flood & Attack 2 & 0.976 & 0.002 & 0.998 & 0.987 \\
UDP flood & Attack 1 & 0.917 & 0.001 & 0.998 & 0.958 \\
& Attack 1 & 1 & 0.005 & 0.995 & 0.998 \\
ICMP & Attack 2 & 1 & 0.001 & 0.999 & 0.998 \\
Smurf & Attack 3 & 1 & 0.001 & 0.999 & 1 \\
& Attack 4 & 1 & 0.001 & 0.999 & 1 \\
\hline
\end{tabular}




\subsection{Detection of MAWI's DOS flooding attacks}

The MAWI database is increasingly attracting researchers and becomes a commonly used dataset in intrusion detection studies. However, this dataset consists of Tcpdump files (pcap format) and providing only the raw traffic network without ground truth. To evaluate our proposed anomaly-based DOS and DDOS detection approach, we have processed the traffic trace to separate the benign (i.e., free from attacks) and anomalous (i.e., attacks) traffic that constitutes the training and testing data, respectively. First, we use the labeling project provided by the MAWI lab to identify attacks and remove them from the traffic trace to construct the training data. Then, the raw Tcpdump files are analyzed to extract different characteristics of such attacks. Table 3 presents the founded information about TCP SYN flood attacks. Fig. 12(a-b) shows the detection results of the proposed ES-KLD scheme in the presence of SYN attacks in the MAWI dataset. In Fig. 12(a), the measurements of the received SYN segments reveal seven instances of attacks (instances: 8, 9, 37, 77, 78, 82, and 83) corresponding to SYN port sweep attacks. At the instance 8, the attacker (@IP: 89.56.8.149) has scanned about 12623 IP address from 208.108.134.x to 208.108.251.x. Table 4 provides the details of these attacks.

Table 3 Syn flood in MAWI Dataset

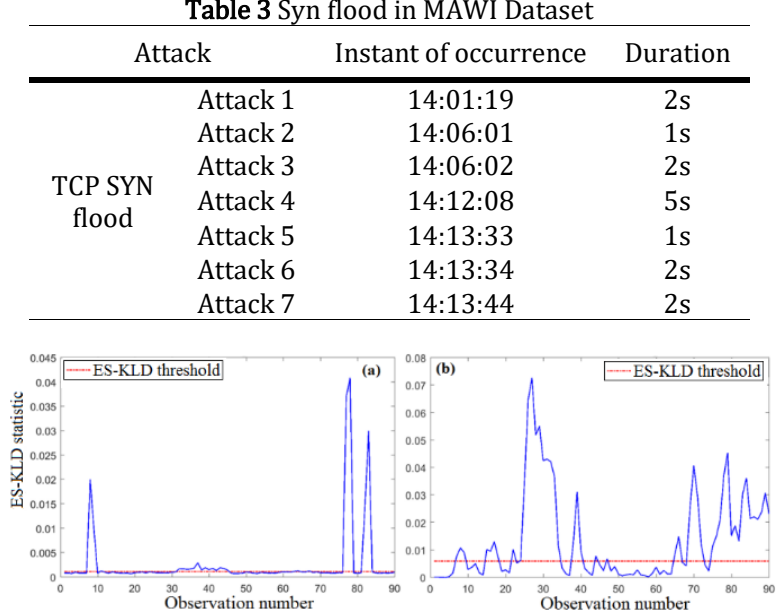

Fig. 12 ES-KLD results in the presence of MAWI SYN flood (a) SYN messages and (b) TCP flow

\subsection{Detection of ICMPv6 traffic's DOS flooding attacks}

The ICMPv6 traffic dataset includes a variety of ICMPv6-based flooding attacks. Here, we investigate the capacity of the ES-KLD mechanism in detecting ICMPv6-based flooding attacks that use the Router Solicitation, Neighbor Solicitation, and Neighbor Advertisement messages. Table 5 summarizes these three types of attacks. Fig.s 13(a-b), 14(a-b), and 15(ab) show the detection results of the ES-KLD mechanism in the presence of ICMPv6-based DOS attacks. As shown in Fig. 13(a), 14(a), and 15(a), the control of Neighbor Advertisement, Neighbor Solicitation, and Router
Advertisement messages have allowed the detection of the considered attacks that are based on such messages. Also, attacks in Fig.s 13(b), 14(b), and 15(b) have been detected by ICMPv6 traffic monitoring Indeed, attacks in this dataset are characterized by high intensity, making them easy to detect. The NAS, NAD, and RAD have very high values in the presence of attacks (i.e., 1681, 2516, and 2591 respectively) compared to attacks-free traffic. Results in Table 6 confirm the capability of the ES-KLD in detecting DOS flooding attacks when using the ICMPV6 dataset.

\begin{tabular}{|c|c|c|c|}
\hline $\begin{array}{l}\text { Attack's } \\
\text { instance }\end{array}$ & Attacker' @IP & $\begin{array}{c}\text { Scannned @IP } \\
\text { plage }\end{array}$ & $\begin{array}{c}\text { Number of } \\
\text { scanned } \\
\text { @IP }\end{array}$ \\
\hline 8 & 89.56.8.149 & $\begin{array}{c}208.108 .134 . x \text { to } \\
208.108 .251 . x\end{array}$ & 12623 \\
\hline 9 & 89.56.8.149 & $\begin{array}{c}208.108 .135 . x \text { to } \\
208.108 .191 . x\end{array}$ & 7875 \\
\hline \multirow{2}{*}{37} & 209.8.83.176 & $\begin{array}{c}209.67 .86 . x \text { to } \\
209.67 .91 . x\end{array}$ & 1312 \\
\hline & 89.56.8.149 & $\begin{array}{c}199.199 .56 . x \text { to } \\
199.199 .63 . x\end{array}$ & 1239 \\
\hline 77 & 93.99.192.106 & $\begin{array}{c}136.172 .128 . x \text { to } \\
136.172 .255 . x\end{array}$ & 18382 \\
\hline 78 & 93.99.192.106 & $\begin{array}{l}\text { 136.172.0.x to } \\
\text { 136.172.235.x }\end{array}$ & 19323 \\
\hline 82 & 200.59 .160 .64 & $\begin{array}{l}209.72 .0 . x \text { to } \\
209.72 .159 . x\end{array}$ & 10243 \\
\hline 83 & 200.59 .160 .64 & $\begin{array}{c}209.67 .64 . x \text { to } \\
209.67 .127 . x\end{array}$ & 16183 \\
\hline
\end{tabular}

Table 5 ICMPV6-based flooding attacks in ICMPv6 traffic dataset

\begin{tabular}{ccc}
\hline Attack & $\begin{array}{c}\text { Instant of } \\
\text { occurrence }\end{array}$ & Duration \\
\hline & & \\
Router advertisement & 1 min $10 \mathrm{~s}$ & $3 \mathrm{~s}$ \\
Neighbor solicitation & $0 \mathrm{~s}$ & $1 \mathrm{~s}$ \\
Neighbor advertisement & $1 \mathrm{~min} 20 \mathrm{~s}$ & $4 \mathrm{~s}$ \\
\hline
\end{tabular}

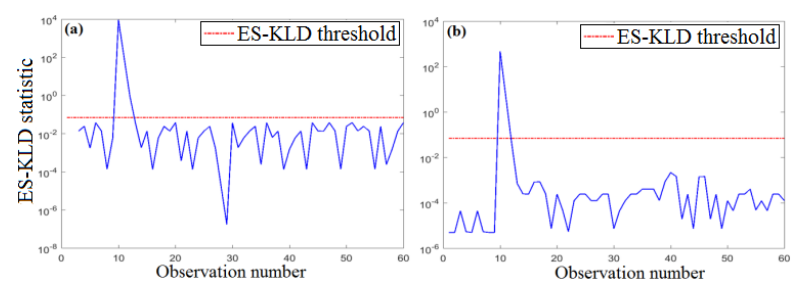

Fig. 13 ES-KLD results in the presence of Neighbor advertisement flood attack,(a) NAD messages, (b) ICMPv6 flow

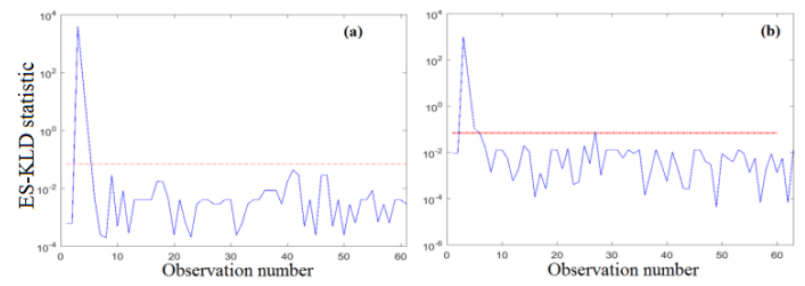

Fig. 14 ES-KLD results in the presence of neighbor Solicitation flood attack (a)NAS messages and (b) ICMPv6 flow 

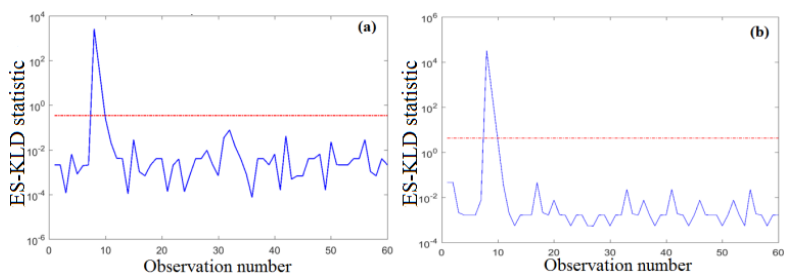

Fig. 15 ES-KLD results in the presence of router advertisement flood attack (a) RAD messages and (b) ICMPv6 flow

Table 6 ES-KLD detection performance when using ICMPV6 dataset

\begin{tabular}{|c|c|c|c|c|c|}
\hline Attack & & TPR & FPR & Accuracy & AUC \\
\hline \multirow{2}{*}{$\begin{array}{c}\text { Neighbor } \\
\text { Advertise-ment }\end{array}$} & $\begin{array}{l}\text { NAD } \\
\text { ICMP }\end{array}$ & 1 & 0 & 1 & 1 \\
\hline & $\begin{array}{l}\text { NAD } \\
\text { flow }\end{array}$ & 1 & 0 & 1 & 1 \\
\hline \multirow{2}{*}{$\begin{array}{l}\text { Neighbor } \\
\text { Solicitation }\end{array}$} & $\begin{array}{l}\text { NAS } \\
\text { ICMP }\end{array}$ & 1 & 0.033 & 0.969 & 0.984 \\
\hline & $\begin{array}{l}\text { NAS } \\
\text { flow }\end{array}$ & 1 & 0.033 & 0.968 & 0.983 \\
\hline \multirow{2}{*}{$\begin{array}{c}\text { Router } \\
\text { Advertisem-ent }\end{array}$} & $\begin{array}{l}\text { RAD } \\
\text { ICMP }\end{array}$ & 1 & 0 & 1 & 1 \\
\hline & $\begin{array}{l}\text { RAD } \\
\text { flow }\end{array}$ & 1 & 0 & 1 & 1 \\
\hline
\end{tabular}

\subsection{Comparison with some state-of-the arts approaches}

Furthermore, Table 7 compares the performance of the ES-KLD mechanism with some state-of-the-art approaches, namely Anomaly Intrusion Detection (AID) [53], Back Propagation Neural Network (BPN) [54], and Support Vector Machine (SVM) [54] when applied to DARPA 99 datasets. The BPN used in [54] is a Feed-Forward Back Propagation Neural Network with two layers and twenty neurons. The SVM in [54] used a fine Gaussian kernel and a one-vs-one multiclass method. Results show that the considered TCP SYN flood attacks were accurately detected using the ESKLD mechanism (i.e., $\mathrm{TPR}=100 \%$ and $\mathrm{FPR}=0.2 \%$ ). Results in Table 8 indicate that the proposed approach outperforms these state-of-the-art methodologies. Table 8 shows that a better detection performance in detecting UDP flood attacks is achieved when using ESKLD and BPN compared to SVM.

Table 7 Comparaison with some DOS appraoches under DARPA 99 Syn flood attack

\begin{tabular}{ccc}
\hline Approach & TPR(\%) & FPR(\%) \\
\hline ES-KLD & 100.0 & 0.18 \\
AID 40x40 [53] & 96.8 & 2.85 \\
AID 30x30 [53] & 96.3 & 3.15 \\
BPN [54] & 99.3 & 0.70 \\
SVM [54] & 99.2 & 0.84 \\
PHAD-C32 [55] & 25.0 & \\
\hline
\end{tabular}

Table 8 Comparaison with some DOS appraoches under DARPA 99 UDP flood attack

\begin{tabular}{lll}
\hline Approach & TPR(\%) & FPR(\%) \\
\hline & & \\
ES-KLD & 100.0 & 0.00 \\
BPN [46] & 100.0 & 0.00 \\
SVM [46] & 98.1 & 0.40 \\
\hline
\end{tabular}

\section{Conclusion}

This paper introduces a reliable KLD-based scheme for DOS and DDOS flooding attacks detection. The method uses the exponentially smoothing KLD (ES-KLD) metric to quantify the deviations of anomalous traffics from the reference (attack-free) traffic. Then, attacks are claimed if the ES-KLD measures exceed a monitoring threshold, computed with a nonparametric approach using kernel density estimation. To validate its effectiveness, this ES-KLD approach is used to detect TCP SYN flood, UDP flood, and ICMPv6 flooding attacks while considering three publically available datasets. Experimental results prove the high performances of ES-KLD. Compared to other solutions, it presents the highest detection rate and reduces false alarms considerably.

Insight of these promising detection results of ESKLD, we intend to extend the present work to other types of DDOS attacks, namely low rate DOS and DDOS (LR-DOS/DDOS) attacks when using real off-line traffic or on an online operational network. On the other hand, to further enhance the performance of ES-KLD in terms of detection accuracy and reduced false alarm rate, we plane to include other traffic features (e.g., acknowledgment (ACK), reset (RST), finished (FIN) TCP segments, and ports) in the monitoring process. To highlight these solutions, we intend to establish a full comparative study with more anomaly-based detection techniques.

In this study, the main challenge is to overcome the system complexity problem and to maintain high detection performances under dynamic changes of traffic features. To alleviate this difficulty, we plan to apply a sliding window to systematically re-set the decision threshold and preserve an accurate detection rate. Recently, deep learning-driven models have emerged as a promising line of research in modeling and forecasting time series data [56, 57]. In future work, we plan to develop advanced cyber-attacks detection techniques by exploiting the extended capacity of deep learning models in modeling time dependencies in time-series data and the sensitivity of the ES-KLD scheme.

Acknowledgement The work presented in this publication was supported by the king Abdullah University of Science and Technology (KAUST) Office of Sponsored Research (OSR) under Award No: OSR-2019-CRG7-3800

\section{References}

1. Hsueh, C.T., Wen, C.Y., Ouyang, Y.C.: A secure scheme against power exhausting attacks in hierarchical wireless sensor networks. IEEE Sens. J. 15(6), 3590-3602 (2015)

2. Miloslavskaya, N., Tolstoy, A.: Internet of Things: information security challenges and solutions. Cluster. Comput. 22,103-119 (2019) 
3. Dhunna, G.S., Al-Anbagi, I. : A low power wsns attack detection and isolation mechanism for critical smart grid applications. IEEE Sens.J. 19(13), 5315-5325 (2019)

4. Zlomislic, A., Fertalj, K., Sruk, V.: Denial of service attacks, defenses and research challenges. Cluster. Comput. 20, 661-671 (2017)

5. Shone, N., Nguyen, N.T., Dinh., P.V., Shi, Q.: A Deep Learning Approach to Network Intrusion Detection. IEEE Trans. Emerg. Top. Comput. Intell. 2(1), 41-50 (2018)

6. Sakhnini, j., Karimipour, H., Dehghantanha, A., Parizi, R.M., Srivastava, G.: Security aspects of Internet of Things aided smart grids: A bibliometric survey. Internet of Things (2019). https://doi.org/10.1016/j.iot.2019.100111

7. Baig, Z. A., Salah, K.: Multi-agent pattern recognition mechanism for detecting distributed denial of service attacks. IET Inf. Secur. 4(4), 333-343 (2010)

8. Bouyeddou, B., Harrou, H., Sun, Y. Kadri, B.: Detecting SYN Flood Attacks via Statistical Monitoring Charts: A Comparative Study. In: Proceeding of the fifth International Conference on Electrical Engineering (ICEE), pp. 1-5. (2017). doi: 10.1109/ICEEB.2017.8192118

9. Harrou, H., Bouyeddou, B., Sun, Y. Kadri, B.: Detecting cyberattacks using a CRPS-based monitoring approach. In: Proceedings of 2018 IEEE Symposium Series on Computational Intelligence (SSCI), pp. 618-622. (2018). doi: 10.1109/SSCI.2018.8628797.

10. Badotra, S., Panda, S.N.: SNORT based early DDoS detection system using Opendaylight and open networking operating system in software defined networking. Cluster Comput. (2020). https://doi.org/10.1007/s10586-020-03133-y

11. Saranya, R., Kannan, S.S., Sundaram, S.M.: Integrated quantum flow and hidden Markov chain approach for resisting DDoS attack and C-Worm. Cluster Comput 22, 14299-14310 (2019). https://doi.org/10.1007/s10586-018-2288-7

12. Fichera, S., Galluccio, L.S., Grancagnolo, C., Morabito, G., Palazzo, S.: Operetta: An openflow-based remedy to mitigate TCP SYN flood attacks against web servers. Comput.Net. 92, 89-100 (2015)

13. Mohammadi, R., Javidan, R., Conti, M.: Slicots: An sdn-based lightweight countermeasure for tcp syn flooding attacks. IEEE Trans. Net.Serv.Manag. 14(2), 487-497 (2017)

14. Sahi, A., Lai, D., Li, Y., Diykh, M.: An efficient DDoS TCP flood attack detection and prevention system in a cloud environment. IEEE Access. 5, 6036-6048 (2017)

15. Velliangiri, S., Premalatha, J.: Intrusion detection of distributed denial of service attack in cloud. Cluster Comput. 22, 10615-10623 (2019). https://doi.org/10.1007/s10586-017-1149-0

16. Saxena, R., Dey, S.:DDoS attack prevention using collaborative approach for cloud computing. Cluster Comput. 23, 1329-1344 (2020). https://doi.org/10.1007/s10586-019-02994-2

17. Bhuvaneswari Amma, N.G., Selvakumar, S.: A statistical class center based triangle area vector method for detection of denial of service attacks. Cluster Comput. (2020). https://doi.org/10.1007/s10586-020-03120-3

18. Kesavamoorthy, R., Ruba Soundar, K.: Swarm intelligence based autonomous DDoS attack detection and defense using multi agent system. Cluster Comput. 22, 9469-9476 (2019). https://doi.org/10.1007/s10586-018-2365-y

19. Wang, C., Yao, H., Liu, Z.: An efficient DDoS detection based on SU-Genetic feature selection. Cluster Comput. 22, 2505-2515 (2019). https://doi.org/10.1007/s10586-018-2275-z

20. Elejla, O.E., Anbar, M., Belaton, B.: ICMPv6-based DoS and DDoS attacks and defense mechanisms, IETE Tech. Rev. 34(4), 390-407 (2017)

21. Olszewski, D.: Fraud detection in telecommunications using kullback-leibler divergence and latent dirichlet allocation. In: Procedings of 2011 International Conference on Adaptive and Natural Computing Algorithms (ICANNGA), pp. 71-80. (2011)

22. Bouyeddou, B., Harrou, H., Sun, Y. Kadri, B.: An Effective Network Intrusion Detection Using Hellinger Distance-Based Monitoring Mechanism. In: Proceedings 2018 International Conference on Applied Smart Systems (ICASS), pp. 24-25. (2018)

23. Harrou, H., Bouyeddou, B., Sun, Y. Kadri, B.: A Method to Detect DOS and DDOS Attacks based on Generalized Likelihood Ratio Test. In: Proceeding 2018 International Conference on Applied Smart Systems (ICASS), pp. 24-25. (2018)

24. Levy-Abegnoli, E., Van de Velde, G., Popoviciu, C., Mohacsi, J. : IPv6 router advertisement guard. Tech. Rep (2011).
25. Bansal, G., Kumar, N., Nandi, S., Biswas, S.: Detection of ndp based attacks using MLD. In: Proceedings of the fifth Internationa Conference on Security of Information and Networks (SIN'12), pp 163-167. (2012)

26. Francois, J., Aib, I., Boutaba, R.: Firecol: a collaborative protection network for the detection of flooding DDoS attacks. IEEE/ACM Trans. Net. 20(6), 1828-1841 (2012)

27. Bouyeddou, B., Harrou, H., Sun, Y. Kadri, B.: Detection of smurf flooding attacks using Kullback-Leibler-based scheme. In: Proceedings of the fourth International Conference on Computer and Technology Applications (ICCTA), pp. 11-15. (2018). doi 10.1109/CATA.2018.8398647.

28. Guo, Z., Shi, D., Johansson, K.H., Shi, L.: Worst-case stealthy innovation-based linear attack on remote state estimation. Automatica, 89, 117-124 (2018)

29. Kung, E., Dey, S., Shi, L.: The performance and limitations of nstealthy attacks on higher order systems. IEEE Trans.Auto.Control. 62(2), 941-947 (2016)

30. Bai, C.Z., Pasqualetti, F., Gupta, V.: Data-injection attacks in stochastic control systems: Detectability and performance tradeoffs, Automatica, 82, 251-260 (2017)

31. Sahoo, K.S., Puthal, D., Tiwary, M., Rodrigues, J.J., Sahoo, B. Dash, R.: An early detection of low rate ddos attack to sdn based data center networks using information distance metrics. Futur. Gen.Comp. Syst. 89, 685-697 (2018)

32. Li, H., Zhang, J., He, X.: Design of data-injection attacks for cyberphysical systems based on kullback-leibler divergence. Neurocomput. $\quad 361, \quad 77-84$ https://doi.org/10.1016/j.neucom.2019.05.085

33. Zhang, Q., Liu, K., Xia, Y., Ma, A. : Optimal stealthy deception attack against cyber-physical systems. IEEE Trans. Cybernetics. (2019). https://doi.org/10.1109/TCYB.2019.2912622

34. Yang, C.:Anomaly network traffic detection algorithm based on information entropy measurement under the cloud computing environment", Cluster. Comput. 22, S8309-S8317 (2019)

35. Rouzbahani, H.M., Karimipour, H., Rahimnejad, A. Dehghantanha, A., Srivastava, G.: Anomaly Detection in CyberPhysical Systems Using Machine Learning. In:Choo, KK. Dehghantanha, A. (eds) Handbook of Big Data Privacy. pp. 219235. Springer, Cham, 2020

36. Mozaffari, F.S., Karimipour, H., Parizi, R.M. : Learning-based anomaly detection in critical cyber-physical systems. In: Choo, K.K.R., Dehghantanha, A (eds) Security of Cyber-Physica Systems, pp. 107-130. Springer, Cham, 2020.

37. Zhiwen, P., Hariri, S., Pacheco, J.: Context aware intrusion detection for building automation systems. Comput. Secur. 85 181-201 (2019)

38. Satam, P., Satam, S., Hariri, S., Alshawi, A.: Anomaly Behavior Analysis of IoT Protocols. Modeling and Design of Secure Internet of Things. pp 295-330 (2020)

39. Zeroual, A., Harrou, F., Sun, Y., Messai, N.: Integrating modelbased observer and kullback-leibler metric for estimating and detecting road traffic congestion, IEEE Sens. J. 18(20), 8605-8616 (2018)

40. Xin, G.: Performance evaluation of automatic object detection with post-processing schemes under enhanced measures in wide-area aerial imagery. Multimed Tools Appl. 79(41), 30357-30386 (2020)

41. Bogdanoski, M., Suminoski, T., Risteski, A.: Analysis of the SYN flood DoS attack, Int. J.Compt. Net.Inf. Secu. 5(8), 1-11 (2013)

42. Gont, F.: Icmp attacks against tcp, Tech. Rep., document RFC 5927 (2010)

43. Singh, N., Agrawal, R.: Combination of kullback-leibler divergence and manhattan distance measures to detect salien objects. Sig. Img. Video Proc, 9(2), 427-435 (2015)

44. Karine, A., Toumi, A., Khenchaf, A., El Hassouni, M.: Target recognition in radar images using weighted statistical dictionarybased sparse representation," IEEE Geo Rem.Sens. Lett. 14(12), 2403-2407 (2017)

45. Harrou, F. Sun, Y, Madakyaru, M. Kullback-leibler distancebased enhanced detection of incipient anomalies. J. Loss Prev.Proc.Industr. 44, 73-87 (2016).

46. Harrou, F., Sun, Y.: Enhanced anomaly detection via PLS regression models and information entropy theory. In: Proceeding of 2015 IEEE Symposium Series on Computational Intelligence (SSCI), pp. 383-388. (2015)

47. Leonard, A. S., Weissman, D., Greenbaum, B., Ghedin, E., Koelle K.: Transmission bottleneck size estimation from pathogen deep- 
sequencing data, with an application to human influenza A virus. J. Vi, JVI-00, 171 (2017)

48. Pardo, L.: Statistical inference based on divergence measures. Chapman and Hall/CRC (2005)

49. Martin, E., Morris, A.: Non-parametric confidence bounds for process performance monitoring charts. J.Proc.Control, 6(6), 349358 (1996)

50. Chen, Y.C.: A tutorial on kernel density estimation and recent advances. Biostat.Epidemi. 1(1), 161-187 (2017)

51. Mugdadi, A. R., Ahmad, I. A.: A bandwidth selection for kernel density estimation of functions of random variables. Comput.Stat.Data Analys. 47(1), 49-62 (2004)

52. Elejla, O. E., Belaton, B., Anbar, M., Alnajjar, A.: A reference dataset for icmpv6 flooding attacks. J. Eng. App.Sciences, 11(3), 476-481 (2016)

53. Zheng, J., Hu, M.: An anomaly intrusion detection system based on vector quantization, IEICE trans. Inf. Syst. 89(1), 201-210 (2006)

54. McDermott, C.D., Petrovski, A.: Investigation of computational intelligence techniques for intrusion detection in wireless sensor networks. Int. J.Comput.Net. Comm.9(4), 45-56 (2017)
55. Mahoney, M., Chan, P.: Packet header anomaly detection for identifying hostile network traffic. In: Proceedings of ACM symposium on applied computing (SAC), pp. 346-350. (2001)

56. Harrou, F., Sun, Y., Hering, A.S. and Madakyaru, M.: Statistical process monitoring using advanced data-driven and deep learning approaches: theory and practical applications. Elsevier (2020)

57. Harrou, F., Hittawe, M.M., Sun, Y. and Beya, O.: Malicious attacks detection in crowded areas using deep learning-based approach, IEEE Inst \& Mea Mag, 23 (5), 57-62 (2020) 